\title{
Access to Systemic Anti-cancer Therapies for Women With Secondary Breast Cancer - Protocol for a Mixed Methods Systematic Review
}

Sally Anne Pearson ( $\nabla$ sally.pearson@postgrad.manchester.ac.uk)

The University of Manchester https://orcid.org/0000-0002-7796-2617

\section{Sally Taylor}

The Christie NHS Foundation Trust

\section{Antonia Marsden}

University of Manchester

Janelle Yorke

The Christie NHS Foundation Trust

\section{Protocol}

Keywords: Access, systemic anti-cancer therapies, secondary breast cancer, inequity unwarranted variation

Posted Date: December 8th, 2020

DOI: https://doi.org/10.21203/rs.3.rs-121889/v1

License: (c) (1) This work is licensed under a Creative Commons Attribution 4.0 International License. Read Full License

Version of Record: A version of this preprint was published at Systematic Reviews on July 23rd, 2021. See the published version at https://doi.org/10.1186/s13643-021-01761-y. 


\section{Abstract}

\section{Background:}

It is well recognised that access and receipt of appropriate guideline recommended treatment with systemic anticancer therapies for secondary breast cancer is a key determinant in overall survival. Where there is disparity in access this may result in unwarranted variation and disparity in outcomes. Individual, clinical and wider contextual factors have been associated with these disparities, however this remains poorly understood for women with secondary breast cancer. The purpose of the review is to address this gap by bringing together the available quantitative and qualitative evidence to examine factors associated with access to systemic anti-cancer therapies for women with secondary breast cancer and explore barriers and facilitators in relation to women and clinicians experience of access.

\section{Methods:}

A mixed methods approach with a segregated design will be used to examine and explore factors which influence access to systemic anti-cancer therapies for women with secondary breast cancer. Quantitative and qualitative appraisal, analysis and syntheses will be conducted discretely prior to final integration of findings. The review will use a comprehensive search strategy to search published and gray literature. Titles and abstracts will be screened against the eligibility criteria and full text articles will be retrieved for all records that meet the inclusion criteria. A data extraction tool will be developed, piloted and refined prior to full data extraction. Methodological quality and risk of bias will be assessed using the Mixed Methods Appraisal Tool.

\section{Discussion:}

Understanding individual, clinical and wider contextual factors associated with access and receipt of systemic anti-cancer therapies for secondary breast cancer is a complex phenomenon. These will be examined to determine any association with access. Review findings will be used to guide future research in this area and the development of an evidence-based service level intervention designed to address unwarranted variation in access based upon the Medical Research Council (MRC) approach to the development, implementation and evaluation of complex interventions.

\section{Systematic review registration:}

The review protocol has been registered in PROSPERO CRD42020196490

\section{Background:}

Equity in access is a global health priority with adequate and fair access being fundamental to the organisation and delivery of high quality health services. Equity of access was considered to be one of the founding principles of the NHS with equitable access to treatment being essential to promote and preserve health. (1). Equity of access is a central component of the UK National Cancer Programme (2) however it is acknowledged that there remains inequity and unwarranted variation in access to treatment across the UK, which disproportionately influences health outcomes $(3,4)$. With regard to cancer treatment it is accepted that access and receipt of appropriate therapy is a key determinant in improving outcomes. Conversely a number of factors have been 
identified as influencing access. However no attempt to date has been made to evaluate factors commonly associated with access to systemic anti-cancer therapies (SACT) for women with secondary breast cancer (SBC).

SBC has been defined as the development of new tumours in tissues and organs away from the primary tumour site (5) with the most common sites of metastases being lungs, liver, bones, and brain. It is estimated that up to $30 \%$ of women with primary breast cancer will experience disease recurrence following initial treatment and despite the increasing effectiveness of treatments, median overall survival is estimated at 3 years with no statistically significant improvement in the past twenty years (6). SBC represents a significant disease burden with high levels of unmet need and unwarranted variation in outcomes (4). Disparities have been reported across the breast cancer continuum which have compromised proper access to and receipt of services, however this remains poorly understood for women with SBC (7). Guideline recommended SACT for the treatment of SBC is intended to optimize patient care informed by the best available evidence and an assessment of the benefits and harms of alternative treatment options. Treatment aims to improve long term survival, increase progression free survival and improve quality of life, though is seldom used with curative intent (8). Current guidelines for SACT treatment for SBC are set out internationally, nationally and regionally and for the purposes of the review guideline recommended SACT are those which have been recommended by National, regional and local bodies.

From a theoretical perspective access has been defined as the "degree of fit between clients and the system" characterised by dimensions of availability, accessibility, accommodation, affordability and acceptability (9). A more contemporary, patient centred framework where multi-level determinants related to health systems, institutions, organisations and providers are considered alongside factors at the individual, household, community, and population levels (10) will provide a theoretical basis for the review.

Review findings will be used to inform the development of an evidence-based service level intervention, based upon the UK Medical Research Council (MRC) approach to the development, implementation and evaluation of complex interventions (11). The intervention will be designed to promote greater standardisation and a reduction in unwarranted variation in access and receipt of treatment.

\section{Review question:}

What individual, clinical and contextual factors influence access to guideline recommended treatment with systemic anti-cancer therapies for women with secondary breast cancer?

\section{Methods And Design:}

The protocol is reported in accordance with the PRISMA-P statement (12) [see additional file 1] and has been registered with the International Prospective Register of Systematic Reviews (PROSPERO) registration number PROSPERO CRD42020196490.

\section{Aim, design and setting:}

The review aims to identify examine and explore factors associated with access to guideline recommended treatment with SACT for women with SBC. It will seek to determine those factors most commonly associated with access and treatment receipt, including those which present barriers and act as facilitators and those which are potentially most amenable to change. 
The review will adopt a mixed methods approach applying a segregated design which will maintain the conventional binary distinction between the qualitative and quantitative paradigm, with integration of each distinct synthesis. The quantitative component aims to identify and investigate those multi-level factors associated with access and receipt of treatment (Table 1.). This will be complimented by the qualitative component which aims to explore how women with secondary breast cancer and their clinicians experience access and receipt of SACT. In particular, to explore barriers and facilitators related to individual, clinical and wider contextual factors relating to health care systems and geographical location. A mixed method approach will be taken as access to treatment is a complex, multi-faceted phenomena which requires a comprehensive, patient centred understanding of how and why and in what context any association exists. This is conducive to a more sophisticated integration of the traditional quantitative and qualitative paradigms offered by a mixed methods approach.

The review setting and context reflects the mixed method approach and will consider studies which explore multilevel factors which have previously been associated with access to treatment. The review will consider studies which investigate and explore access to SACT, including chemotherapy, targeted therapy, immunotherapy and hormone/endocrine therapy. This will include studies which report across secondary, tertiary, specialist and palliative care centres, where SACT is accessed as either, first, second or subsequent line of treatment.

\section{Study eligibility:}

The review will consider studies that include women aged 18 and over with an SBC diagnosis who have accessed and received (or otherwise) treatment for their disease with SACT. This will include studies reporting all clinical sub types of SBC and all sites of metastases. The study population will be consistent across quantitative and qualitative components.

Table 1

Multi-level factors associated with access and receipt of treatment.

\begin{tabular}{|c|c|}
\hline Factors & Characteristics \\
\hline \multirow[t]{6}{*}{ Individual factors: } & - Age, gender, sexual orientation \\
\hline & - Race/ethnicity \\
\hline & - Socioeconomic status \\
\hline & - Education \\
\hline & - Language and literacy \\
\hline & - Psychosocial characteristics \\
\hline \multirow{3}{*}{$\begin{array}{l}\text { Clinical } \\
\text { characteristics: }\end{array}$} & - Clinical sub type, including, Hormone receptor status, HER2 status \\
\hline & - Previous treatment response \\
\hline & - Physician characteristics \\
\hline \multirow[t]{2}{*}{$\begin{array}{l}\text { Contextual } \\
\text { factors: }\end{array}$} & $\begin{array}{l}\text { - Geography and geographical location, Including, distance, travel time, health region, } \\
\text { catchment/referral areas }\end{array}$ \\
\hline & - Organisational Including, health care system factors, capacity, service availability \\
\hline
\end{tabular}


Studies reporting the primary outcome measure of access to and receipt of SACT as either, first, second or third line treatment for secondary breast cancer will be included. The review will be limited to studies published in peer reviewed journals which report original research in English language from January 2000 onwards. Quantitative studies will include observational studies, cross sectional, longitudinal and analytic studies, including, epidemiological studies, case control and cohort studies. RCTs reporting access and receipt will be included though where they report safety and efficacy alone, will be excluded. Qualitative studies will include designs such as phenomenology, grounded theory, ethnography, action research and feminist research. Descriptive qualitative studies that describe the experience or the effects of the experience will also be considered. Table 2 presents a summary of study inclusion and exclusion criteria. 
Table 2

Summary of study inclusion and exclusion criteria.

Study inclusion criteria

Studies reporting on women aged 18 and over with a confirmed secondary breast cancer diagnosis who accessed treatment with a systemic anti-cancer therapy for their disease. This will include studies reporting all clinical sub types across all sites of metastases and will include de novo presentations.

\section{Study exclusion criteria}

Studies reporting on women with a primary diagnosis of early stage (IIII) breast cancer only

Studies reporting on women with a diagnosis of locally advanced breast cancer (LABC) with local or loco regional recurrence only

Studies measuring (comparative) treatment effect, efficacy and studies reporting clinical trials of systemic anti-cancer therapies, as whilst RCT is not a specific exclusion criteria, it is likely that RCTs will measure treatment effect as opposed to access as an outcome and would therefore be excluded.

Males with a secondary breast cancer diagnosis, as this is classified as a rare disease

\section{Phenomena of interest:}

Intervention/Exposure:
Studies which explore factors that are associated with access to SACT for SBC.

Studies reporting on access and receipt of systemic anti-cancer treatment at secondary, tertiary or specialist cancer centres and units. This will include a diverse range of health care organisations and systems.

\section{Outcome(s):}

Studies reporting the primary outcome measure of access to, and utilization of systemic anti-cancer therapies. This will include studies reporting access to chemotherapy, single agent or combination, hormone/endocrine therapy, targeted therapy and immunotherapy, as either, first, second or third line treatment, for secondary breast cancer.
Studies which do not report the primary outcome measure of access to, and utilization of systemic anti-cancer therapies 


\begin{tabular}{|c|c|c|}
\hline & Study inclusion criteria & Study exclusion criteria \\
\hline Types of study: & $\begin{array}{l}\text { The review will consider quantitative, } \\
\text { qualitative and mixed methods studies. This } \\
\text { will include observational, cross sectional, } \\
\text { longitudinal and analytic studies, including, } \\
\text { epidemiological studies, case control and } \\
\text { cohort studies. } \\
\text { Qualitative studies will include designs such } \\
\text { as phenomenology, grounded theory, } \\
\text { ethnography, action research and feminist } \\
\text { research. Descriptive qualitative studies that } \\
\text { describe the experience or describe the } \\
\text { effects of the experience will also be } \\
\text { considered. Mixed method studies will only } \\
\text { be considered if data from the quantitative or } \\
\text { qualitative components can be clearly } \\
\text { extracted. } \\
\text { Studies published in English language, from } \\
\text { January } 2000 \text { onwards, peer reviewed, } \\
\text { original research, reporting quantitative, } \\
\text { qualitative or mixed methods }\end{array}$ & $\begin{array}{l}\text { Studies measuring (comparative) } \\
\text { treatment effect, efficacy and } \\
\text { studies reporting clinical trials of } \\
\text { systemic anti-cancer therapies, as } \\
\text { whilst RCT is not a specific } \\
\text { exclusion criteria, it is likely that } \\
\text { RCTs will measure treatment effect } \\
\text { as opposed to access as an } \\
\text { outcome and would therefore be } \\
\text { excluded. }\end{array}$ \\
\hline
\end{tabular}

\section{Search strategy and information sources:}

A modified PICO framework was used to develop the review question (13). This was then used to formulate the search strategy by identifying key concepts, subject headings and keywords (Table 3). A preliminary search was undertaken to identify articles on the topic with text words in titles and abstracts of relevant articles. This was then used to develop a full search strategy. The search strategy including all identified keywords and index terms will be tailored to each information source. The electronic databases to be searched will be The Cochrane Library, EBSCO CINAHL Plus, Ovid MEDLINE, Ovid EMBASE, PsychINFO. Reference lists of included studies will also be screened. The basic search strategy will be tailored to individual databases and undertaken with the support of an experienced librarian/evidence specialist. Grey literature will be searched using NHS Evidence. 
Table 3

Basic search strategy, key concepts and search terms.

\begin{tabular}{|c|c|c|c|c|}
\hline PICO: & Population & Exposure & Outcome & Study design \\
\hline $\begin{array}{l}\text { Key } \\
\text { concept: }\end{array}$ & $\begin{array}{l}\text { Secondary } \\
\text { breast } \\
\text { cancer }\end{array}$ & $\begin{array}{l}\text { Factors which } \\
\text { influence access }\end{array}$ & $\begin{array}{l}\text { Systemic anti-cancer therapies } \\
\text { (guideline recommended) }\end{array}$ & $\begin{array}{l}\text { Quantitative, } \\
\text { qualitative, mixed } \\
\text { methods }\end{array}$ \\
\hline $\begin{array}{l}\text { Subject } \\
\text { headings } \\
\text { and } \\
\text { keywords: }\end{array}$ & $\begin{array}{l}\text { Secondary } \\
\text { OR } \\
\text { advanced } \\
\text { OR } \\
\text { metastat* } \\
\text { Breast OR } \\
\text { mammary } \\
\text { Cancer OR } \\
\text { neoplasm } \\
\text { OR tumour } \\
\text { OR } \\
\text { oncolog* }\end{array}$ & $\begin{array}{l}\text { Factors: } \\
\text { sociodemographics } \\
\text { OR socioeconomic } \\
\text { ethnicity OR race } \\
\text { education OR } \\
\text { sexual orientation } \\
\text { psycho* (social) } \\
\text { geograph* OR } \\
\text { location OR } \\
\text { distance } \\
\text { clinical } \\
\text { characteristics, } \\
\text { hospital and } \\
\text { physician } \\
\text { characteristics } \\
\text { Access: } \\
\text { Access OR health } \\
\text { care OR } \\
\text { accessibility OR } \\
\text { utilisation }\end{array}$ & $\begin{array}{l}\text { aromatase inhibitors OR } \\
\text { tamoxifen OR hormone therapy } \\
\text { OR oestrogens OR epidermal } \\
\text { growth factors } \\
\text { Biological/targeted therapy: } \\
\text { Antibodies, monoclonal OR } \\
\text { biological therapy OR } \\
\text { methotrexate } \\
\text { Immunotherapy: } \\
\text { Immunotherapy }\end{array}$ & $\begin{array}{l}\text { RCT, cohort study, } \\
\text { observational study, } \\
\text { case control study, } \\
\text { epidemiological } \\
\text { study, survey, case } \\
\text { series } \\
\text { Phenomenology, } \\
\text { ethnography, } \\
\text { grounded theory, } \\
\text { interview, focus group } \\
\text { Mixed methods }\end{array}$ \\
\hline
\end{tabular}

\section{Study screening and selection:}

Records will be stored and managed in EndNote X9. Titles and abstracts will be screened against the inclusion and exclusion criteria. Potentially eligible studies will be retrieved in full. Full text articles will then be assessed against the inclusion and exclusion criteria, with reasons for exclusion recorded and reported. Screening and full text assessment will be undertaken by two independent reviewers with any disagreement resolved through discussion. Where consensus cannot be reached this will be achieved through arbitration with a third reviewer. The results of the search will be reported in full in the final report and presented in a Preferred Reporting Items for Systematic Reviews and Meta-analyses (PRISMA) flow diagram (14).

\section{Data extraction:}

Data will be extracted using an adaptation of a standardized data extraction tool which will be piloted prior to commencement of full data extraction. Extracted data will reflect the review question, reported as identified in the included studies to reduce the risk of introducing bias. It will include details of study population, setting, context, phenomena of interest, methodology and methods, multi-level factors associated with access and receipt of 
treatment and the primary outcome. Main findings will be extracted and assigned a level of credibility based upon methodological quality and risk of bias.

\section{Assessment of methodological quality and risk of bias:}

Methodological quality of included studies will be assessed using the Mixed Methods Appraisal Tool (MMAT). (15) This will be undertaken by two independent reviewers with any disagreement resolved through discussion or where required with arbitration from a third reviewer. This process will be followed for quantitative and qualitative studies. The results of critical appraisal will be reported in narrative form accompanied by a summary table. Risk of bias for included studies will be classified as low risk, high risk, or unclear (either lack of information or uncertainty over the potential for bias). The Cochrane Collaboration tool for assessing risk of bias will be used for included randomised controlled trials and the preliminary risk of bias in non-randomised studies - of exposures (ROBINS-E) tool will be used for non-randomised interventional studies. $(16,17)$. Studies will not be excluded based upon low methodological quality or credibility, however this will be reflected in the analysis and synthesis.

\section{Data analysis, synthesis and integration:}

The review will follow a segregated approach to analysis and synthesis. This will involve distinct quantitative and qualitative analysis and synthesis followed by integration of the resultant evidence. Analysis and synthesis of quantitative included studies will be presented in narrative form together with tables and figures to aid data presentation. The narrative will be structured around multi-level factors which have shown an association with access and receipt of treatment as the primary outcome measure for the review. This will be determined a priori guided by the theoretical model of access adopted to guide the review (10). Factors associated with access and receipt will be classified as individual, clinical and contextual. Where possible data will be pooled using statistical meta-analysis. This will be undertaken in accordance with guidance for meta-analysis of observational studies where appropriate. Heterogeneity will be assessed using the standard chi squared and I2 tests (18). Analyses will be performed using a random effects model to estimate a summary measure of access and treatment receipt for each factor, with an overall summary measure of the likelihood of receipt of treatment for each included study. Qualitative findings will be presented in narrative form using a narrative synthesis approach (19). Where possible these will be pooled using meta-aggregation to produce a comprehensive set of synthesized qualitative findings. Final synthesis of quantitative and qualitative findings will combine the separate synthesis into a set of conclusions which will reflect the narrative synthesis of qualitative findings and the quantitative findings produced from meta-analysis (where appropriate) to configure a mixed research synthesis.

\section{Discussion:}

Understanding multi-level factors associated with access and receipt of SACT for SBC is a complex phenomenon. Examining these factors in a comprehensive way will enable the development of evidence based strategies to address the challenges which they may present. It is acknowledged that appropriate access is a key determinant in overall survival, yet disparities have compromised proper access to and receipt of services resulting in unwarranted variation in outcomes. However, the extent of these disparities, the underlying reasons and the impact on outcomes remains poorly understood. A number of theoretical frameworks have been proposed to conceptualise access. The multi-level factors and complexity of SACT treatment pathways for SBC led to the selection of a patient centred model of access to guide the review. The model was selected as it provided a contemporary perspective on access based on a synthesis of the published literature. The model incorporated 
dimensions of accessibility which represent supply, which when integrated with the corresponding abilities of persons to interact, generate access. Thus the review will address those individual and clinical characteristics which influence the ability of individuals to interact to create access alongside dimensions of accessibility relating to wider contextual factors, including organisational and health care systems factors.

The complexity of the review question meant this could be most appropriately answered using a mixed methods approach, in particular a segregated design where qualitative and quantitative findings are viewed distinctly as complementary and synthesis is configurative. There are potential challenges in accurately and consistently identifying and quantifying multi-level factors which may be associated with access. From a methodological perspective this will be addressed using narrative synthesis and where appropriate, sub group analysis for each factor to determine the extent and significance of the association. This will be further explored in the qualitative analysis and brought together through the synthesis. The inclusion of observational studies considerably expands the challenges in establishing a level of inference with a greater risk of confounding resulting from selection bias. Additionally, including only references published in English could lead to selection and reporting bias, which will be addressed through the analysis. In summary, the importance and significance of answering the review question outweighs the balance of the potential limitations. The impact of these have been reflected in the methodology and will be accounted for and addressed in the analysis, synthesis and integration of findings.

\section{List Of Abbreviations:}

MRC Medical Research Council

MMAT Mixed Methods Appraisal Tool

SACT: Systemic Anti-Cancer Therapies

SBC: Secondary Breast Cancer

\section{Declarations:}

\section{Ethics approval and consent to participate:}

Not applicable.

\section{Consent for publication:}

Not applicable.

\section{Availability of data and materials:}

Not applicable

\section{Competing interests:}

The authors declare that they have no competing interests.

\section{Funding}


The review is being undertaken as part of a PhD fellowship funded by The Christie Hospital NHS Foundation in collaboration with the Manchester Cancer Research Centre (MCRC) at The University of Manchester.

\section{Authors' contributions:}

SP: Lead author

ST: Academic supervision and proof reading

AM: Statistical advice and proof reading

JY: Lead academic advisor

Acknowledgements:

Professor Andrew Wardley

\section{References:}

1. The NIHR Evaluation, Trials and Studies Coordinating Centre: Access to Health Care - Report of a Scoping Exercise for the National Co-ordinating Centre for NHS Service Delivery and Organisation R \& D (NCCSDO) http://www.netscc.ac.uk/hsdr/files/project/SDO_FR_08-1009-005_V01.pdf (2001). Accessed: 12 May 2020

2. NHS England: Achieving World-Class Cancer Outcomes: A Strategy for England 2015-2020 Progress Report 2016-17.(2017) https://www.england.nhs.uk/wp-content/uploads/2017/10/national-cancer-transformationprogramme-2016-17-progress.pdf Accessed 3 June 2020

3. NHS England: Compendium Atlas of Variation. https://www.england.nhs.uk/rightcare/products/atlas/ (2016). Accessed 2 September 2020

4. All Party Parliamentary Group on Breast Cancer: A Mixed Picture: An Inquiry into Geographical Inequalities and Breast Cancer. https://breastcancernow.org/get-involved/campaign-us/all-party-parliamentary-groupbreast-cancer/report-geographical-inequalities-in-breast-cancer (2018). Accessed 28 August 2020

5. National Cancer Institute: Metastatic cancer https://www.cancer.gov/types/metastatic-cancer (2020) Accessed 3 August 2020

6. Metastatic Breast Cancer Network: 2020) Incidence and incidence rates http://mbcn.org/incidence-andincidence-rates/ (2020). Accessed: 7 May 2020

7. Cardoso F, Spence D, Mertz S, et al. Global analysis of advanced/metastatic breast cancer: Decade report (2005-2015). The Breast. 2018; doi: https://doi.org/10.1016/j.breast.2018.03.002

8. National Institute for Health and Care Excellence: Managing Advanced Breast Cancer file:///C:/Users/sally/Downloads/advanced-breast-cancer-managing-advanced-breast-cancer\%20(13).pdf (2020) Accessed 10 October 2020

9. Penchansky R, Thomas J. The Concept of Access: Definition and Relationship to Consumer Satisfaction. Medical Care. 1981; doi: https://www.jstor.org/stable/3764310

10. Levesque J, Harris MF, Russell G. Patient-centred access to health care: conceptualising access at the interface of health systems and populations. International Journal of Equity Health. 2013; doi: http://doi.org/10.1186/1475-9276-12-18 
11. Medical Research Council: Developing and evaluating complex interventions. https://mrc.ukri.org/documents/pdf/complex-interventions-guidance/ (2008). Accessed: 18 August 2020

12. Moher D, et al. Preferred reporting items for systematic review and meta-analysis protocols (PRISMA-P) 2015 statement. Systematic Reviews. 2015; doi: https://doi.org/10.1186/2046-4053-4-1

13. Joanna Briggs Institute: JBI Manual for Evidence Synthesis. https://wiki.jbi.global/display/MANUAL/JBI+Manual+for+Evidence+Synthesis (2020). Accessed 4 August 2020

14. Moher D, et al. Preferred reporting items for systematic reviews and meta-analyses: the PRISMA statement. BMJ. 2009; doi: https://doi.org/10.1136/bmj.b2535

15. Mixed Methods Appraisal Tool: The Mixed Methods Appraisal Tool - version 2018. http://mixedmethodsappraisaltoolpublic.pbworks.com/w/file/139355532/MMAT_2018_criteria_WORD_201808-08.docx Accessed 23 August 2020

16. Higgins JPT, Savović J, Page MJ, Elbers RG, Sterne JAC. Chapter 8: Assessing risk of bias in a randomized trial. In: Higgins JPT, Thomas J, Chandler J, Cumpston M, Li T, Page MJ, Welch VA (editors). Cochrane Handbook for Systematic Reviews of Interventions version 6.1 (updated September 2020). 2020. https://training.cochrane.org/handbook/current/chapter-08. Accessed 26 August 2020

17. Centre for Research Synthesis and Decision Analysis: The ROBINS-E tool (Risk Of Bias In Non-randomized Studies - of Exposures) https://www.bristol.ac.uk/media-library/sites/social-community medicine/images/centres/cresyda/Risk_of_bias_preliminary_for_exposures_template_Jul2017.pdf (2017). Accessed 1 November 2020

18. Deeks JJ, Higgins JPT, Altman DG (editors). Chapter 10: Analysing data and undertaking meta-analyses. In: Higgins JPT, Thomas J, Chandler J, Cumpston M, Li T, Page MJ, Welch VA (editors). Cochrane Handbook for Systematic Reviews of Interventionsversion 6.1 (updated September 2020). 2020. https://training.cochrane.org/handbook/current/chapter-10. Accessed 26 August 2020

19. Thomas J, Harden A. Methods for the thematic synthesis of qualitative research in systematic reviews. Biomed Central Medical Research Methodology. 2008; doi: https://doi.org/10.1186/1471-2288-8-45

\section{Supplementary Files}

This is a list of supplementary files associated with this preprint. Click to download.

- ADDITIONALFILE1CompletedPRISMAChecklist.docx

- ADDITIONALFILE1CompletedPRISMAChecklist.docx

- ADDITIONALFILE1CompletedPRISMAChecklist.docx 